\title{
An Analysis on Scheduling of Traffic Light at Urban Traffic Intersection Using Fuzzy Control Algorithm
}

\author{
Ma Wen, Burra Venkata Durga Kumar \\ School of Information Science and Technology, Xiamen University Malaysia, Sepang District, Malaysia \\ Email address: \\ CST1709284@xmu.edu.my (Ma Wen), Venkata.burra@xmu.edu.my (B. V. D. Kumar)
}

\section{To cite this article:}

Ma Wen, Burra Venkata Durga Kumar. An Analysis on Scheduling of Traffic Light at Urban Traffic Intersection Using Fuzzy Control Algorithm. International Journal of Transportation Engineering and Technology. Vol. 7, No. 3, 2021, pp. 85-91.

doi: $10.11648 /$ j.jitet.20210703.14

Received: September 7, 2021; Accepted: October 4, 2021; Published: October 19, 2021

\begin{abstract}
In modern society, private cars have become the first choice for many families because of their convenience and versatility. The volume of vehicles on the road is the basis of traffic accident and traffic congestion. In urban sector the traffic congestion is normally high due to the green light time interval at four road intersections. The traffic light control and time setting are basically timer control operation at current traffic control management system, this shows that, the current system is not intelligent so that there is still heavy traffic congestion. It is vital to implement routinely adjusted schedule as per the real-time position of vehicles at urban cross road intersection. Now, there are various detector systems for traffic monitoring, like Inductive Loop microwave radar, laser, infrared, ultrasonic, magnetometer and video image processing. But they have relevant weakness, such as high cost and complex technology. As a more and more widely used technology, image processing plays an important role in the management and control of intelligent transportation system. Image processing systems are based on motion detection of vehicles, wherein computer vision algorithms extract vehicles from traffic video data for traffic density estimations. This paper is an analysis on scheduling of traffic light of traffic management system using Fuzzy Control Algorithm. With the increase of the number of vehicles and population, it will also improve the traffic jam and the mood of people because of the cause of jam. Rather than previous technology, it will be low cost and simple, which can be adopted in every place as far as possible. MATLAB tool was used to figure out the variables impact on scheduling of traffic light at urban traffic intersection. The vehicle number, vehicle speed, lane length and vehicle type variables are identified and tested against vehicle driving for conclusion on traffic management performance. From findings the results were identified as the vehicle number, vehicle speed, and vehicle type have significant positive relationship with vehicle driving. However, the lane length did not significantly affect the vehicle driving. This indicates that the lane length is less important in scheduling of traffic light at urban traffic intersection.
\end{abstract}

Keywords: Fuzzy Control Algorithm, Vehicle Number, Vehicle Speed, Lane Length, Vehicle Type, Vehicle Driving, Scheduling

\section{Introduction}

In modern society, private cars have become the first choice for many families because of their convenience and versatility. Urban sectors traffic congestion is not only due to urban sectors growth, but also due to the current traffic control system technology. At many places still the cross road intersections traffic control signal lights are controlled by timer system. With the rapid development of intelligent algorithms, researchers have applied intelligent control algorithms to the field of traffic information control [1]. The commonly used intelligent control algorithms include fuzzy control algorithm and artificial neural network control algorithm. And this research will propose a way to determine the scheduling of traffic light based on fuzzy control algorithm, for which the input is queue length and queue difference and the output is the time of green time. Through the simulation, traffic congestion has improved to a large degree.

Traffic signals have been utilized to schedule and manage traffic at every intersection, which regulates the competing traffic flows with light cycle schedules. They offer secured scheduling that licenses all traffic movements to communicate the road intersection [2]. And the traffic flow fluidity is influenced by the queuing delay at each road 
intersection, which consequently reduces traffic capacity at each intersection [3]. For a phase, the vehicles in the lane are moving dynamically in a certain direction, but the signal light does not change.

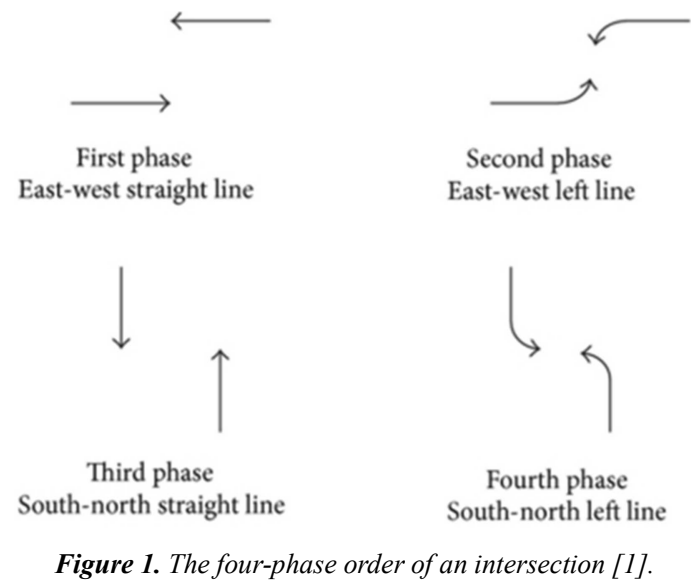

Figure 1 shows that the traffic intersection system of four-phase is vital to study the traffic condition [4]. In the traffic light system running under the conventional timing strategy, each phase time is fixed, while under the intelligent control strategy, the phase time will change according to the demand. And the signal cycle will change [4]. The commonly used intelligent control algorithms include fuzzy control algorithm and artificial neural network control algorithm [5]. The Back Propagation (BP) learning algorithm of BP network is the most commonly used neural network algorithm [6] The development of fuzzy cybernetics may be traced back to this groundbreaking study [5].

\subsection{Fuzzy Control Algorithm}

In the field of traditional control, most of the control algorithm as a testing index is to judge the algorithm and inferiority, but large number control systems have too many arguments, the corresponding mathematical model is difficult to establish. In order to simplify the model, many experts apply mathematics to the control system, and fuzzy control is a kind of nonlinear control system [7].

Fuzzy control is a control approach that makes use of fuzzy mathematics' core principles and theories. The more complete the system dynamic information is; the more precise control may be performed in the conventional control field. To access the fuzzy control toolbox in Matlab, type fuzzy and then two-dimensional fuzzy PID controller. After fuzzy, anti-fuzzification and fuzzy rules inference, the output can be got [7].

\subsection{Fuzzy Relation and Fuzzy Reasoning}

Fuzzy relation matrix R elements are Fuzzy control rules, which are constructed with number of conditional statements. The fuzzy vector $\mathrm{E}$ is the driving force to cybernetics domain from matrix $\mathrm{R}$ domain, also the fuzzy vector $\mathrm{E}^{\prime}$ of the bias and the deviation of the fuzzy vector EC', the fuzzy vector of the control quantity is obtained by fuzzy reasoning with the synthesis of the fuzzy relation matrix R, and then the fuzzy control vector is converted to the precise quantity by anti-fuzzification method. According to fuzzy set and fuzzy relation theory, different fuzzy inference methods can be used for different types of fuzzy rules [8].

\subsection{Fuzzy Decision of Fuzzy Control Vector}

The control quantity obtained from the above is a fuzzy set, and the anti-fuzzification method is needed to convert the fuzzy control item into an exact quantity. There are two simple and practical methods.

(1) Maximum membership degree method

In this method, the control quantity with the major relationship degree is selected as the output of the fuzzy controller.

(2) Weighted average decision method

In order to overcome the disadvantages of the determined relationship method, the weighted average decision method can be used [8].

\subsection{Fuzzy Control Table}

The operation of fuzzy relation, fuzzy reasoning and fuzzy decision can be carried out offline. Finally, the quantitative level of fuzzy controller input is obtained. The deterministic relationship between deviation change and output quantity, namely the quantitative level of system control quantity, is usually called the control table. The fuzzy control table can be worked out offline and stored in the computer as a file. When the computer controls in real time, it only needs to quantify the deviation and deviation change obtained from conversion to obtain the corresponding grade, and then directly query the control strategy needed to be taken from the file [8].

\section{Problem Statement}

Traditional traffic lights use a defined period to regulate traffic. Even if there are no vehicles on the road, there will be a period of waiting. In reality, traditional systems are incapable of dealing with a variety of situations since they can only respond to the same traffic volume distribution situations in all directions. If it is determined which direction should have a longer green light signal period in traffic, it may be predicted that needless traffic light waiting time will be avoided. And the time of traffic light can also be depended on by traffic volume on the road [9]. In order to improve traffic efficiency, researchers have proposed intelligent algorithms to scheduling of traffic lights [1]. The optimal scheduling of every traffic light minimizes average delay time of vehicles at urban intersections. The traffic control of each traffic light is an indefinite series time period, which represents the time scheduling of each traffic flow that will pass through a certain road intersection $[8,10,11]$.

One-dimensional fuzzy controller has poor dynamic performance and is usually used for first-order controlled objects. Two-dimensional fuzzy controller has better control performance and complexity, which is a widely used form at 
present. And the incremental algorithm is usually chosen as the output variable of the fuzzy controller [7, 12]. Fuzzy control is language control, so it is necessary to use language to summarize the expert's manual control strategy, so as to establish the fuzzy control rule table. Manual control policies can generally be described with conditional statements.

PTV VISSIM Functionalities:

PTV VISSIM is a microscopic multi-modal traffic flow simulation software package developed by PTV Planung Transport Verkehr AG in Karlsruhe, Germany. The name is derived from Verkehr In Städten SIMulations modell (PTV VISSIM), which is the ideal tool for state-of-the-art transportation planning and operations analysis. The software is designed to assist users in realistically simulating and balancing roadway capacity as well as traffic and transport demand. Signal control system with high performance is one of the key functions. Some ESSG (External Signal State Generator) software, such as VS-PLUS, TRENDS, and other applications, can be utilized with PTV VISSIM. VISSIM can also be utilized in combination with existing signal controllers when the interface is called signal control system [7, 13].

\section{Research Objectives}

1. To study traffic basic parameters.

2. To study fuzzy control algorithm.

3. To design road model using PTV VISSIM and test.

4. To identify the relationship between factors.

\section{Research Questions}

1. Does vehicle numbers factor affect vehicle driving?

2. What extent the vehicle speed factor affect vehicle driving?

3. Does vehicle lane length factor affect vehicle driving?

4. Does vehicle type factor affect vehicle driving?

\section{Framework}

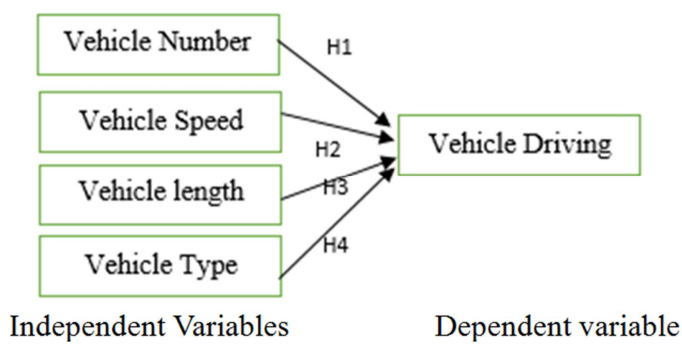

Figure 2. Framework.

Figure 2 shows research framework with independent variables and dependent variable; the hypotheses are:

1. $\mathrm{H}_{0}$ : There is no significant relationship between vehicles number and vehicle driving.

2. H1: There is a significant relationship between vehicles number and vehicle driving. The more cars on the road, the more likely it is to cause traffic jam.

3. $\mathrm{H} 2_{0}$ : There is no significant relationship between vehicle speed and vehicle driving

4. H2: There is a significant relationship between vehicle speed and vehicle driving. Generally speaking, the faster vehicle travels on a road, the more capacity the road will have. According to the regulations, the maximum speed on the expressway is $120 \mathrm{~km} / \mathrm{h}$.

5. $\mathrm{H} 3_{0}$ : There is no significant relationship between lane length and vehicle driving.

6. H3: There is a significant relationship between lane length and vehicle driving. The longer the lane length, the longer the traffic time.

7. $\mathrm{H}_{0}$ : There is no significant relationship between vehicle type and vehicle driving.

8. H4: There is a significant relationship between vehicle type and vehicle driving. The larger vehicles there are, the more likely it is to block traffic

\section{Methodology}

The paper is about an analysis on scheduling of traffic light based Fuzzy Control Algorithm, and the goal is to improve traffic congestion through decreasing average delay time and average number of stops. With the increase of the number of vehicles and population, it will also improve the quality of people's life and the mood of people because of the cause of jam. Rather than previous technology, it will be more intelligent and efficiency, which can be adopted in every place as far as possible. In this research we used the quantitative method, which uses proposed membership assignment table of the fuzzy variable by expert. And the paper will calculate the time of green light according to the table of fuzzy variable using MATLAB, for which the input of fuzzy controller is queue length and queue length difference and the output is the time of green light. The PTV VISSIM software was used to assess the scheduling of traffic light, which calculates related average delay time and average number of stops and compares with traditional traffic light to analyze average delay time. In order to compare the relationship between the factors and traffic driving, the paper also uses Control variable method to analyze average delay time. The project will set same data for other variables from the analysis of the relationship between vehicle volume and driving method data.

\subsection{Setting up Traffic Model}

This project adopts traffic intersection system of four-phase, which there are east-west straight line, east-west left line, south-north straight line, south-north left line respectively

\subsection{Setting up Parameters}

In order to fully analyze the advantages and disadvantages of the algorithm, this project uses PTV VISSIM to model and simulate the intersection, which adopts Wiedemann 74 driving behaviors and the simulation time of each state is $600 \mathrm{~s}$. Besides, the same number of vehicles is adopted for each phase. The traffic parameters observed in the simulation 
mainly include the number of vehicles and the speed of vehicles passing through traffic intersections.

\subsection{Running a Cycle in Fixed Model}

In daily life, the signal light needs to enter the yellow light for a period of time after the green light ends and before the red light starts, because the vehicles crossing the stop line during the yellow light can pass the intersection. In order to simplify the model, the yellow light time is set to $3 \mathrm{~s}$ in this project. And for the sake of testing and evaluating the designed fuzzy controller, this project guarantees the same simulation time, and uses the traditional timing control and the intelligent control to conduct the simulation respectively.

\subsubsection{Setting the Queue Length and Queue Difference}

The queue length and queue difference are the input of the system to get optimized green light time.

\subsubsection{Calculating the Green Light Time}

The timing scheme of intelligent control is obtained after the traffic parameters obtained by simulation are calculated by the fuzzy control module of MATLAB.

\subsubsection{Process Using Fuzzy Control Algorithm (MATLAB)}

Urban traffic system is a complex system of strong randomness and fuzziness, which is an accurate model cannot be established for traffic intersection according to the number of cars $[14,15]$. And fuzzy control algorithm does not require the mathematical model of project object, which is suitable to signal control. The key part of the fuzzy control is fuzzy rules that are determined by human. In this system, the input and output are blurred in a certain range, which reduce the complex degree of algorithm to satisfy the requirement of real-time and optimal control [16].

Fuzzy control algorithm is a special intelligent control algorithm, which simulates human fuzzy logic thinking to control signal. And fuzzy control theory is based on fuzzy mathematics, which the exact input quantity will be transformed into fuzzy quantity through mathematics fuzzy relation in fuzzy reasoning basis. Then according to the pre-selected membership function, the fuzzy input after transformation is inferred to the corresponding fuzzy output. Finally, the fuzzy output is transformed into the actual output according to a certain fuzzy algorithm.

Table 1. Membership assignment table of fuzzy variable $L$.

\begin{tabular}{|c|c|c|c|c|c|c|c|c|c|c|c|}
\hline \multirow{2}{*}{$\mathbf{L V}$} & \multicolumn{11}{|c|}{ Queue length } \\
\hline & 2 & 4 & 6 & 8 & 10 & 12 & 14 & 16 & 18 & 20 & 22 \\
\hline VS & 1 & 0.6 & 0.1 & 0 & 0 & 0 & 0 & 0 & 0 & 0 & 0 \\
\hline S & 0.2 & 0.7 & 1 & 0.7 & 0.2 & 0 & 0 & 0 & 0 & 0 & 0 \\
\hline LS & 0 & 0.2 & 0.8 & 1 & 0.8 & 0.2 & 0 & 0 & 0 & 0 & 0 \\
\hline M & 0 & 0 & 0 & 0.1 & 0.6 & 1 & 0.6 & 0.1 & 0 & 0 & 0 \\
\hline LL & 0 & 0 & 0 & 0 & 0 & 0.2 & 0.8 & 1 & 0.8 & 0.2 & 0 \\
\hline $\mathrm{L}$ & 0 & 0 & 0 & 0 & 0 & 0 & 0.2 & 0.7 & 1 & 0.7 & 0.2 \\
\hline VL & 0 & 0 & 0 & 0 & 0 & 0 & 0 & 0 & 0.1 & 0.6 & 1 \\
\hline
\end{tabular}

Table 1 shows each phase key queue length $\mathrm{L}$ (The greater the length of two opposing lane queues in a phase) $\mathrm{L}$ as fuzzy variables, the theory of domain as: $L=\{2,4,6,8,10,12,14$,
16, 18, 20\}. VS (very short), S (short), LS (little small), M (medium), LL (little long), L (long), and VL (very long), LV (linguistic values).

Table 2. Membership assignment table of fuzzy variable $G$.

\begin{tabular}{|c|c|c|c|c|c|c|c|c|c|c|c|}
\hline \multirow{2}{*}{$\mathbf{L V}$} & \multicolumn{11}{|c|}{ Queue difference } \\
\hline & -10 & -8 & -6 & -4 & -2 & $\mathbf{0}$ & 2 & 4 & 6 & 8 & 10 \\
\hline NB & 1 & 0.6 & 0.1 & 0 & 0 & 0 & 0 & 0 & 0 & 0 & 0 \\
\hline $\mathrm{NM}$ & 0.2 & 0.7 & 1 & 0.7 & 0.2 & 0 & 0 & 0 & 0 & 0 & 0 \\
\hline NS & 0 & 0.2 & 0.8 & 1 & 0.8 & 0.2 & 0 & 0 & 0 & 0 & 0 \\
\hline $\mathrm{ZO}$ & 0 & 0 & 0 & 0.1 & 0.6 & 1 & 0.6 & 0.1 & 0 & 0 & 0 \\
\hline PS & 0 & 0 & 0 & 0 & 0 & 0.2 & 0.8 & 1 & 0.8 & 0.2 & 0 \\
\hline PM & 0 & 0 & 0 & 0 & 0 & 0 & 0.2 & 0.7 & 1 & 0.7 & 0.2 \\
\hline PB & 0 & 0 & 0 & 0 & 0 & 0 & 0 & 0 & 0.1 & 0.6 & 1 \\
\hline
\end{tabular}

Table 3. Membership assignment table of fuzzy variable $R$.

\begin{tabular}{|c|c|c|c|c|c|c|c|c|c|c|c|}
\hline \multirow{2}{*}{$\mathbf{L V}$} & \multicolumn{11}{|c|}{ Green light time } \\
\hline & 2 & 4 & 6 & 8 & 10 & 12 & 14 & 16 & 18 & 20 & 22 \\
\hline VS & 1 & 0.6 & 0.1 & 0 & 0 & 0 & 0 & 0 & 0 & 0 & 0 \\
\hline S & 0.2 & 0.7 & 1 & 0.7 & 0.2 & 0 & 0 & 0 & 0 & 0 & 0 \\
\hline LS & 0 & 0.2 & 0.8 & 1 & 0.8 & 0.2 & 0 & 0 & 0 & 0 & 0 \\
\hline M & 0 & 0 & 0 & 0.1 & 0.6 & 1 & 0.6 & 0.1 & 0 & 0 & 0 \\
\hline LL & 0 & 0 & 0 & 0 & 0 & 0.2 & 0.8 & 1 & 0.8 & 0.2 & 0 \\
\hline $\mathrm{L}$ & 0 & 0 & 0 & 0 & 0 & 0 & 0.2 & 0.7 & 1 & 0.7 & 0.2 \\
\hline VL & 0 & 0 & 0 & 0 & 0 & 0 & 0 & 0 & 0.1 & 0.6 & 1 \\
\hline
\end{tabular}


Table 2 shows The current phase of queue difference as the second fuzzy variables, the theory of domain is: $(\mathrm{G}=\{-10,-8$, $-6,-4,-2,0,2,4,6,8,10\})$. NB (negative big), NM (negative medium), ZO (negative zero), PS (positive small), PM (positive middle), PB (positive large), LV (linguistic values).

Similarly, the green light time is blurred and its domain is: $\mathrm{G}=\{2,4,6,8,10,12,14,16,18,20,22\}$. VS (very small), $\mathrm{S}$ (small), LS (little small), M (medium), LL (little long), L (long), VL (very long), LV (linguistic values) see table 3.

Table 4 shows The fuzzy controller in this project has two inputs, and there are 7 fuzzy subsets of queue length and queue length difference respectively. Total of 49 fuzzy rules need to be designed.

Table 4. Membership assignment table of fuzzy variable $G$.

\begin{tabular}{|c|c|c|c|c|c|c|c|}
\hline \multirow{2}{*}{ QL } & \multicolumn{7}{|c|}{ Queue difference } \\
\hline & NB & NM & NS & ZO & PS & PM & PB \\
\hline VS & S & S & S & VS & VS & VS & VS \\
\hline S & $\mathrm{S}$ & S & S & $\mathrm{S}$ & VS & VS & VS \\
\hline LS & LS & LS & LS & LS & $\mathrm{S}$ & S & $\mathrm{S}$ \\
\hline M & M & M & M & $\mathrm{M}$ & LS & LS & LS \\
\hline LL & LL & LL & LL & M & M & M & M \\
\hline $\mathrm{L}$ & $\mathrm{L}$ & $\mathrm{L}$ & $\mathrm{L}$ & $\mathrm{L}$ & LL & LL & LL \\
\hline VL & VL & VL & VL & VL & VL & $\mathrm{L}$ & $\mathrm{L}$ \\
\hline
\end{tabular}

QL: Queue Length

\subsubsection{Resolve the Fuzzy Output}

After importing the set fuzzy rules into the fuzzy system, a three-dimensional demonstration of the input and output relations of the fuzzy system can be obtained:

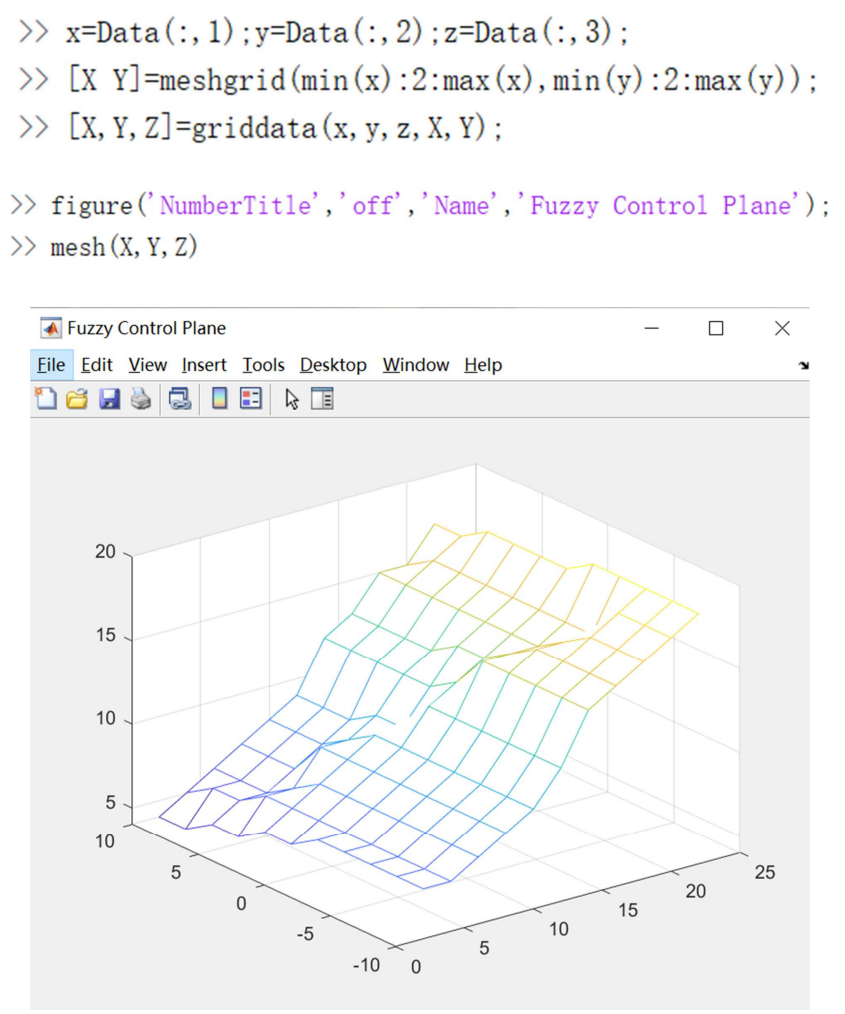

Figure 3. Fuzzy Control Plane.

Figure 3 Fuzzy Control Plane explains three-dimensional plane between the input and output. $X$ axis represents queue length, $\mathrm{Y}$ axis represents queue difference, and $\mathrm{Z}$ axis represents the time of green light.

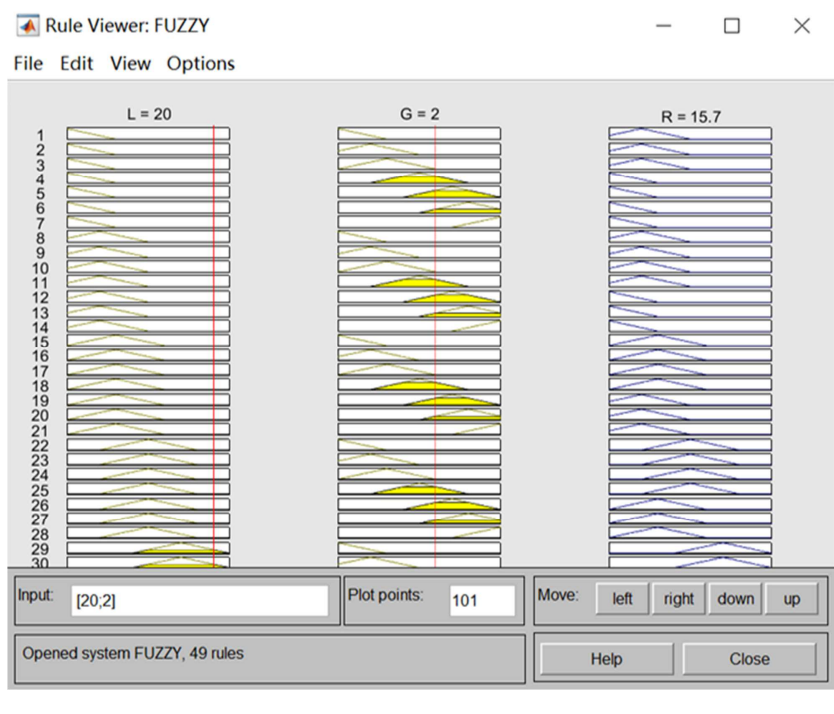

Figure 4. Output.

Figure 4 shows L represents queue length, $G$ represents queue difference, $\mathrm{R}$ represents green light time.

\section{Findings}

Figure 5 shows the value of average delay time before and after optimization. $\mathrm{X}$ axis represents the volume from 100 to 1500 which each increment is $100.1-15$ is the digit as per vehicle numbers, which each number corresponding a value of volume. Y represents average delay time.

Figure 6 shows the value of average number of stops before and after optimization. $\mathrm{X}$ axis represents the volume from 100 to 1500 which each increment is $100.1-15$ is the digit as per raw data, which each number corresponding a value of volume. $\mathrm{Y}$ represents average number of stops. 


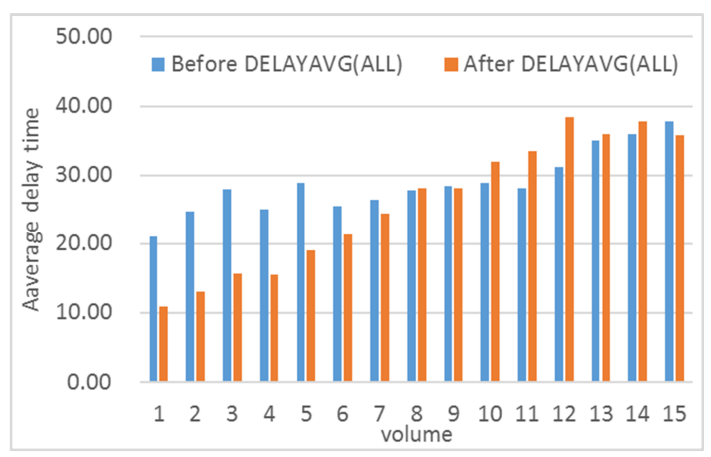

Figure 5. Average delay time before and after optimization.

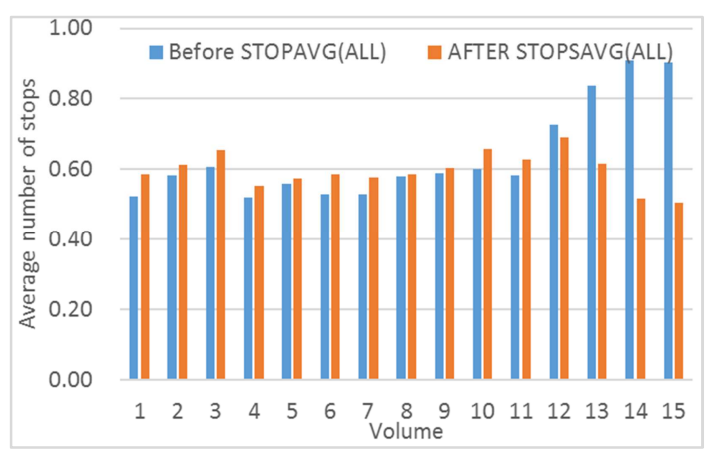

Figure 6. Average delay time before and after optimization.

Asper vehicle speed is $5 \mathrm{~km} / \mathrm{h}-120 \mathrm{~km} / \mathrm{h}$. For the other variables, the project adopts same data. And Before Delay_avg and before Stop-avg represent average delay time and average number of stops when the traffic model runs in fixed model. Similarly, After Delay_avg and After Stop_avg refer average delay time and average number of stops after using the fuzzy control algorithm.

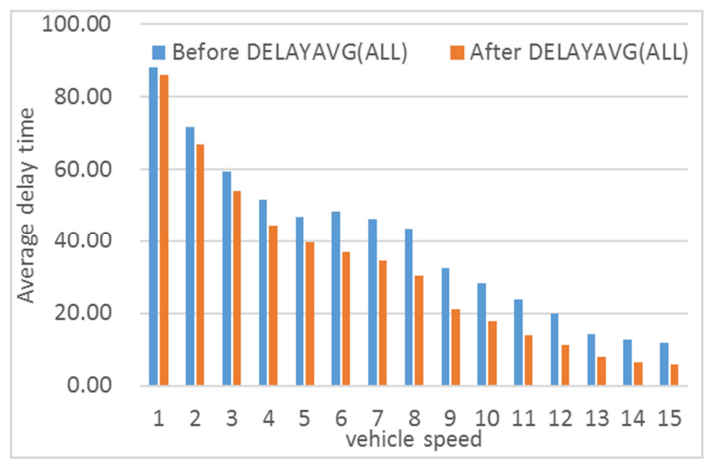

Figure 7. Average delay time before and after optimization.
Figure 7 shows the value of average delay time before and after optimization. $\mathrm{X}$ axis represents vehicle speed from 5 $\mathrm{km} / \mathrm{h}$ to $120 \mathrm{~km} / \mathrm{h} .1-15$ is the digit as per vehicle speed data, which each number corresponding a speed $\mathrm{Y}$ axis refers to the value of average delay time.

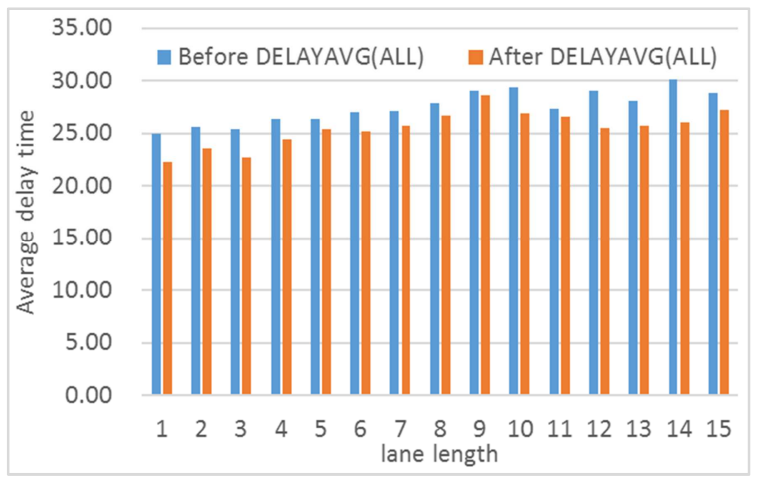

Figure 8. Average delay time before and after optimization.

Figure 8 shows the value of average delay time before and after optimization. $\mathrm{X}$ axis represents lane length from 75.63 to $147.58 \mathrm{~m} .1-15$ is the digit as per lane length data, which each number corresponding a value of lane length. $\mathrm{Y}$ axis refers to the value of average delay time.

Figure 9 shows the value of average delay time before and after optimization. $\mathrm{X}$ axis represents vehicle type from car only to trunk only. 1-15 is the digit as per vehicle type, which each number corresponding a proportion of traffic flow. Y axis refers to the value of average delay time.

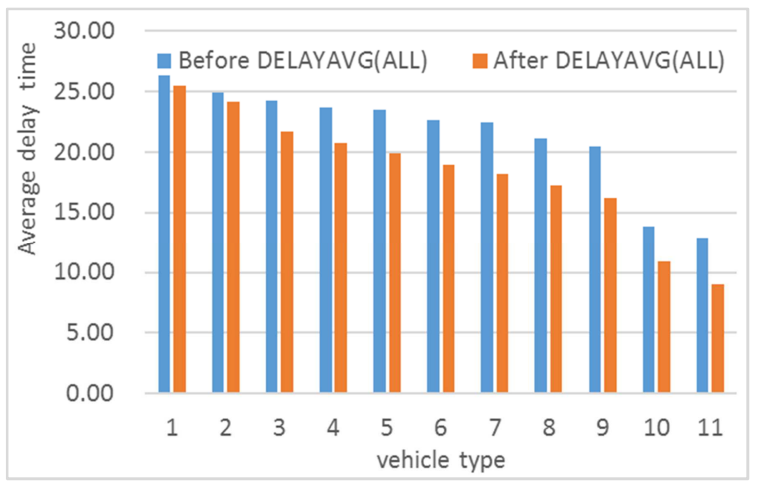

Figure 9. Average delay time before and after optimization.

Table 5. Results.

\begin{tabular}{lll}
\hline Hypo & Description & Status \\
\hline H1 & There is a significant positive relationship between vehicle number and vehicle driving. \\
H2 & There is a positive significant relationship between vehicle speed and vehicle driving. \\
H3 & There is no significant relationship between lane length and vehicle driving. & Accepted \\
H4 & There is a significant positive relationship between vehicle type and vehicle driving & Accepted \\
H4 & There is a significant positive relationship between vehicle type and vehicle driving. & Rejected \\
\hline
\end{tabular}

As the vehicle volume increases, so does the average delay and the number of stops. In the case of small volume, the optimization effect of average delay time is the most obvious, the vehicle speed increases, the average delay time and the number of stops decrease, which represent increased capacity on the road. In the case of high volume, the optimization effect 
of average delay time is the most obvious, the lane length increases, the average delay and the number of stops do not change sharply compared to the first two variables. Similarly, the optimization effect for delay time and stopping times is not influenced by increasing lane length. In general, the algorithm can improve traffic jams to some extent, the proportion of trunk increases, the average delay and the number of stops increase. However, the optimization effect for delay time and stopping times is gradually enhanced, which shows that this algorithm is more suitable for high vehicle size as shown in Table 5.

\section{Conclusion}

According to the results of traffic intersections with different flow rates, different vehicle speeds, different lane lengths and different vehicle types, it can be seen that the fuzzy control algorithm designed in this research can improve traffic congestion on the whole, and the optimization effect of average delay time is more obvious than the average number of stops. As the traffic flow gradually increases, the average delay time and the average number of stops both increase successively, which also indicates the serious traffic jam on the road. Under different flow parameters, the degree of optimization is not the same. In the low traffic flow condition, almost all the four phase times can completely release the queuing waiting vehicles, so the number of stops is relatively small. However, at high-flow intersections, ordinary timing strategies cannot adjust the length of phase green time according to the queue length and queue difference of vehicles, so sometimes queuing vehicles cannot pass through the intersection at one time, resulting in longer stopping time and times of stops. However, the fuzzy control algorithm can adjust the green time according to the real-time traffic parameters. When there are more vehicles, the passage time will be increased, so the stop time and the number of stops under the fuzzy control will be optimized, hence there is significant positive relationship between vehicle number, vehicle speed, vehicle type and vehicle driving. Because average delay time and average number of stops do not have obvious change with the increasing lane length, there is no significant relationship between lane length and vehicle driving. As a more and more widely used technology, image processing plays an important role in the management and control of intelligent transportation system. Now, there are many various detector systems for traffic monitoring, like Inductive Loop Microwave RADAR, LASER, Infrared, Ultrasonic, Magnetometer and Video image processing. These technologies are useful for future research work in intelligent transportation system.

\section{References}

[1] N. Kumar, N. Anand Singh, R. Pal, and M. Kumar Sharma, "Automated traffic management system using image Processing". International Journal of Advanced Research in Electronics And Communication Engineering (IJARECE), 2017. 6 (4).
[2] H. Zhao, G. Han, and X. Niu, "The signal control optimization of road intersections with slow traffic based on improved PSO". Mob. Netw. Appl. 2019, 1-9. \%2Fs11036-019-01225-7.

[3] M Younes,., and A Boukerche, "An efficient dynamic traffic light scheduling algorithm considering emergency vehicles for intelligent transportation systems". Wireless Networks, 2017. 24 (7), 2451-2463. doi: 10.1007/s11276-017-1482-5.

[4] C. Jin, W. Wang, and R. Jiang, "Four-phase or two-phase signal plan? A study on four-leg intersection by cellular automaton simulations". International Journal of Modern Physics C, 2016. 27 (03), 1650032. doi: 10.1142/s0129183116500327.

[5] N. Kumar, S. Rahman, and N. Dhakad, "Fuzzy Inference Enabled Deep Reinforcement Learning-Based Traffic Light Control for Intelligent Transportation System". IEEE Transactions on Intelligent Transportation Systems, 2020. 1-10. doi: 10.1109/tits.2020.2984033.

[6] G. Weiwei, "Research on scheduling algorithm of intelligent traffic light". Microcomputer Application, 2017), (TP301.6; U491.51).

[7] C. Urrea, J. Kern, and J. Alvarado "Design and Evaluation of a New Fuzzy Control Algorithm Applied to a Manipulator Robot", Applied Sciences, MDPI, 2020 10, 7482; doi: 10.3390/app10217482.

[8] J. Jiang, Z. Wang, and F. Chen, "Urban Traffic Signals Timing at Four-Phase Signalized Intersection Based on Optimized Two-Stage Fuzzy Control Scheme”, Hidawi, 2021, Volume 2021, Article 6693562, https://doi.org/10.1155/2021/6693562.

[9] M. Razavi, M. Hamidkhani, and R. Sadeghi "Smart Traffic Light Scheduling in Smart City Using Image and Video Processing". Conference: 2019 3rd International Conference on Internet of Things and Applications (IoT). 2019.

[10] S. Zhu, Y. Zhao, Y. Zhang, Q. Li, W. Wang, and S. Yang, "Short- term traffic flow prediction with wavelet and multi-dimensional taylor network model," IEEE Transactions on Intelligent Transportation Systems, 2020, vol. 99, pp. 1-6.

[11] B. L. Ye, W. Wu, K. Ruan, L. Li, T. Chen, H. Gao, and Y Chen, "A survey of model predictive control methods for traffic signal control”. IEEE/CAA J. Autom. Sin. 2019, 6, 623-640.

[12] J. Lin, J. Zhou, M. Lu, H. Wang, and A. Yi, "Design of robust adaptive fuzzy controller for a class of single-input single-output (siso) uncertain nonlinear systems," Mathematical Problems in Engineering, 2020. vol. 2020, no. 9, Article ID 6178678, 11 pages.

[13] J. C. Chedjou, and K. Kyamakya, A review of traffic light control systems and introduction of a control concept based on coupled nonlinear oscillators. Recent Adv. Nonlinear Dyn. Synchronization 2018, 109, 113-149.

[14] T. Jiang, Z. Wang, and F. Chen, "Urban Traffic Signals Timing at Four-Phase Signalized Intersection Based on Optimized Two-Stage Fuzzy Control Scheme. Math”. Probl. Eng. 2021.

[15] S. Jafari, Z. Shahbazi, Y. C. Byun, "Improving the Performance of Single-Intersection Urban Traffic Networks Based on a Model Predictive Controller". Sustainability 2021, 13, 5630. https://doi.org/10.3390/su13105630. 